\title{
MEANING OF MOMENT OF LIFE IN A COMPLEX WORLD - A POST-PANDEMIC STUDY
}

\author{
Mohamed Buheji \\ International Inspiration Economy Project, Bahrain
}

\begin{abstract}
The paper tests the influence of the application of the 'meaning of the living moment' in a complex, and unstable world. Studies reviewed show that without meaning, individuals and communities are more likely to fail to capture the profound knowledge that any complexity could bring.

The implication of this study is that it addresses a missing element that is not well addressed when dealing with or planning to deal with the rising mental health issues, that is realising the essence and the meaning of the moment during challenging times. The conclusion shed a new path that could lead to the enhancement, readiness and availability of a more agile generation that turns the suffering into sources of hardiness and tolerance through the emphasis on the meaning or purpose of living. This could even turn what is seen as sources of suffering into niches for new opportunities.
\end{abstract}

Key words: Meaning, Complex Problems, Suffering, Meaning of the living Moment, Mental Health, COVID-19 Pandemic

Cite this Article: Mohamed Buheji, Meaning of Moment of Life in a Complex World - A Post-Pandemic Study, International Journal of Management (IJM), 12(9), 2021, pp. 32-42.

https://iaeme.com/Home/issue/IJM?Volume=12\&Issue=9

\section{INTRODUCTION}

In this paper, we review the challenges of meaning loss and the importance of finding meaning and a purpose in life, especially during unprecedented times as during and post COVID-19 pandemic. The importance of this work is that it comes during persistent pandemic waves that bring more halt or disruption to a complex world. Therefore, the researcher seeks to understand how finding or re-shaping 'meaning for the moment of life' at this generation-defining moment could overcome the negative consequences of this multiloading complexity.

The literature illustrates how complexity increases instability, especially in socioeconomic environments, and gives coping mechanisms during unprecedented times. The researcher goes through the constructs needed for a meaningful life and how to utilise the earlier work of Buheji and Ahmed (2020 a, b) that focus on seeing opportunities during the crisis. The framework of inspiration economy for exploitation of opportunities out of complex problems during 
unprecedented times is used as an analogy for meaning development. Sources of meaning development are illustrated to overcome the impairment caused by stressful events that caused anxiety. The paper test influence of the application of the inspiration economy framework on meaning re-shaping and sharpening. The conclusion brings sheds a new path on maintenance of meaning that could help to enhance readiness and availability of essence of living during challenging times, which could alleviate suffering in a complex world.

\section{LITERATURE REVIEW}

\subsection{Defining a 'Meaning for the Moment of Life'}

Personal meaning can be defined as a collection of purpose-driven values that create personal significance and satisfaction (Wong, 1989). Personal meaning for the essence of existence or role in life is found to be an important source for wellbeing and a protective factor against mental health problems. Meaning is found to play a role in reconciling the discrepancy between the factual situation and the previously held beliefs or assumptions, as per Park (2010).

Enhancement of meaning found to improve both health and the therapeutic efforts, besides sets the beliefs to better reflects the suffering and can enhance meaning, largely by providing coherence, Van Tongeren and Showalter (2021). When people find meaning, they find connections with both themselves and others. They begin then to feel that they are significant and that they matter, regardless of the uncomfortable reality around us.

Meaning found to reduce death-related anxiety and death-thought accessibility makes this life more meaningful, Buheji (2020b). With the global COVID-19 pandemic, a call for a better understanding of meaning effects and how it can flourish people and community life is becoming more important, and in fact, very essential. In the midst of this crisis, meaning for existence needs to be re-shaped or maintained. Latest studies show that lack of meaning was found to be related to psychological distress, Ho et al. (2010), Steger and Frazier (2005).

Wong (2016) seen that personal meaning can overcome any source of suffering, and could lead to flourishment in life purpose regardless of circumstances. Meaning found to help raise readiness to confront stressful situations and alleviates distress. With meaning, the mindset would be prepared to see the positive side of the challenges and consider them as part of living experiences.

\subsection{Re-Shaping Meaning to Enhance Tolerance and Alleviates Suffering}

Suffering is considered the main source of existential anxiety that impairs one's ability to find meaning. By cultivating or re-shaping meaning, we address suffering and could raise resilience capacity, Edwards and Van Tongeren (2020).

Effective processing of social information and the navigation of social relationships help to re-shape meaning and manage potential existential anxiety, da Silva et al. (2020). Restoring meaning reduces suffering by directly addressing existential concerns and mitigating suffering. Edwards and Van Tongeren (2020). Edwards and Van Tongeren (2020) found that when meaning is low, the suffering intensity was found to be high. i.e., suffering was associated with lower meaning which included not sharpen life purposefulness, and not enough coherence. The loss of profound meaning was found to mediate the relationship between suffering and wellbeing, de Jong et al. (2020).

Rebuilding meaning by acceptance of existential frailty can create new assumptions and renew the mindset perspectives about the reality of the world. Once the mindset is a renewed sense of coherence can be accepted, Martela and Steger (2016). Van Tongeren et al. (2015) mentions that redefining meaning can be done through re-establishing a sense of relational connection that can help reduce suffering. Relationships are often a primary source of meaning 
in life, and when people reengage in social relations with others, such as through relational repair behaviours as forgiveness, people report greater meaning in life. Stillman et al. (2009), Heintzelman and King (2014).

Going through a sudden crisis, or tragedy as the death of loved ones, or losing the source of income, or losing secure life can often be a type of suffering that can shack up the dimensions of meaning and make the world does not make sense anymore. Testoni et al. (2018). Edwards and Van Tongeren (2020) seen suffering had significant associations with personal meaning, mental health, and wellbeing. Furthermore, the personal meaning was significantly associated with mental health and wellbeing and mediated the negative effect of suffering on mental health and wellbeing. Schnell and Krampe (2020), Steger and Frazier (2005).

Hardiness and psychological strengths were found to be highly related to meaning in life, resilience, and hope, as per Buheji (2020c). Arslan and Allen (2021) investigated the mediating effect of personal meaning on COVID-19 pandemic related suffering and mental health problems that influenced life satisfaction among the Turkish population.

\subsection{Constructs of Meaningful Life}

A large survey revealed multiple differing predictors of happiness (controlling for meaning) and meaningfulness (controlling for happiness). Satisfying one's needs and wants increased happiness but was largely irrelevant to meaningfulness, Hooker et al. (2020). Happiness was largely present-oriented, whereas meaningfulness involves integrating past, present, and future. For example, thinking about the future and past was associated with high meaningfulness but low happiness. Higher levels of worry, stress, and anxiety were linked to higher meaningfulness but lower happiness, Buheji et al. (2020c). Concerns with personal identity and expressing the self-contributed to meaning but not happiness. We offer brief composite sketches of the unhappy but meaningful life and of the happy but meaningless life. Buheji (2020d).

Building meaning through developing healthy relationships can not only help overcome the existential anxiety of concerns, reduce the feeling of isolation, and improve the wellbeing, Ho et al. (2010). When people build meaning through connections to religion and spirituality, they address the stress of existential concerns. This helps them to transcend towards more flourishing lives. Buheji et al. (2020c), Steger and Frazier (2005).

Heine et al. (2006) affirm that humans are natural meaning-makers; they look to develop coherence, feel significant, and have a life purpose that permeates their daily experiences as part of their psychological functioning. However, their meaning can be only cultivated and reshaped by challenges, or stress, or hardship. Baumeister et al. (2013). Another study by Buheji and Jahrami (2020) found that people with differentiated hardiness are different in their ability to find meaning in life. Acceptance and profound realisation of reality shape and rebuild meaning for the remaining time of life and help people to be more rational with new sharper perspectives.

\subsection{Importance of Finding Meaning during COVID-19 Pandemic}

Van Tongeren and Green (2018) confirms that meaning is found to be the central feature of human flourishing. Building meaning across all domains should lead people to be more life purposeful and focus on a richer and healthy life. The persistence of length of the COVID-19 pandemic brought many challenges that were enlarged by the lengthy isolation and consistent reminders of death, Testoni et al. (2018). This led to a collective feeling of meaninglessness among many people after months of fear of a pandemic, Buheji (2020d). Many people are struggling to find coherence, feel of significance, or understand the purpose of why they are continuing life, and this even increases their challenges to their own meaning, de Jong et al. (2020). Many are feeling more senseless the more they struggle to come to terms with a 
seemingly random shared trauma and grief. This once-in-a-generation disruptive event has challenged many to what has been concrete or a reliable purpose. Buheji (2020), Martela and Steger (2016).

A recent longitudinal study by VanderWeele (2020) revealed that meaning in life had been impaired significantly when compared to the pre-COVID-19 pandemic, which might have a drastic effect on mental health and social functioning. Stillman et al. (2009). Cultivating meaning during this ongoing pandemic is a primary way to address these concerns and their concomitant anxiety. Trzebinski et al. (2020) found that greater meaning in life managed to reduce anxiety and stress during the COVID-19 pandemic.

\subsection{Importance of Meaning in a Complex World}

Complex systems, by contrast, are imbued with features that may operate in patterned ways but whose interactions are continually changing, Buheji (2017). Due to living in an ever-increasing complex world, Van Tongeren and Showalter (2021) found that suffering elicits existential anxiety, which impairs the collective sense of meaning. The increase in complexity make people feel senseless or being challenged for their coherence. Such feeling makes people question whether they matter, which lead to undermining the meaning of living with purposefulness. Schnell and Krampe (2020).

Increasing complexity in a networked world can create instability and could be beyond control. It may not be possible to understand this ahead of time. This inability to understand and manage systemic risk is an important challenge for current risk assessments, including in the context of the response to the COVID-19 pandemic. Buheji (2020a), Buheji and Ahmed (2021), Buheji and Ahmed (2020a).

The world is going towards more complexity as the sources of the problems are coming from a variety of reasons, especially those related to socio-economic issues. The increase in the subspecialties and the emphasis of the capital economy-based solution drives towards a closed scarcity mindset. Therefore, the current and future world problems complexity requires both holistic multidisciplinary thinking and approaches. This would transform the way we deal with the complicated problems, i.e., the problems that need time \& financial resources, but are not necessarily complicated. Buheji (2020d)

\subsection{Uniqueness of Socio-economic Projects Complexity}

When you have a complicated socioeconomic issue, the outcomes can be predicted by knowing the input conditions of the project; however, all it needs is a maturity time to be solved. However, in a complex socioeconomic system, what matters is the different outcomes produced from the same issue. Therefore, the complexity comes and increase with the interactions of the elements in the system. Allen et al., (2011).

Complex socioeconomic issues require the minimization of friction between all the constructs of the issues. This type of organic system needs to be seen from different perspectives if we tackle its formula. This means we need diverse thinking approaches to deal with the changes and variations that will inevitably occur. Besides, multidisciplinary thinking we also need a mindset that would work on mitigation of risks when dealing with both complicated and complex issues, so that early failures can be kept to the minimum.

Sometimes to avoid bias in observations collected for major socioeconomic complex problems, multi-different background observers who are clear about their life meaning need to be involved. The complexity of the socioeconomic issues emphasizes interactions through feedback loops, and these feedback loops could be enlarged with multidisciplinary approaches and help to bring radical changes. Complexity characterises the behaviour of a system and helps 
to create a model that interacts in multiple ways that enhance the possibility for different interactions that could bring solutions greater than the sum of its parts.

\subsection{Meaning and Coping with Complexity}

'Meaning of the living moment' is found to enhance our capacity for coping with the complex environment as it supports being more available to the disruptive environment and improves our visibility with the processes and outcomes. Our capacity to deal with diverse perspectives when making decisions help to improve our coping with the gaps created by the different sources of disruptions caused by the unintended consequences we experience suddenly. The meaning of living moments would make our coping remains to be measurable. Buheji and Ahmed (2020a).

Heine et al. (2006) found that meaning creates a central motivation for human coping, as it carries within meaning-making mechanisms that are represented through self-esteem, certainty, and a sense of belonging. The strength of coping or responses to sources of anxiety or threats was found to be highly related to the ability to relate to the meaning of the moment. This ability to have a term of reference to why we are suffering and how to deal with this moment, helps to regain psychological equanimity that occurs beyond conscious awareness (Van Tongeren and Green, 2010).

Park (2010) emphasis that meaning-making at the right moment helps to cope with stress and adversity, which leads to discrepancy between the way people expect the world to be and their assessment of the particular event (i.e., the difference between the global vs. the situational meaning). The more the coping is done the moment, the less distress we could experience. Coping with distress mitigates any possibility for a sense of impairment in the meaning of life. Park (2010), Van Tongeren and Showalter (2021). This mechanism of coping and capacity to mitigate any possible impairment found that have more opportunity to control the poorer mental health or quality of wellbeing caused by the spillovers of the COVID-19 pandemic.

Park (2010) has seen even during a hostile environment, similar to the current pandemic, the process of meaning-making allows one to cope through modifying existing beliefs, values, and assumptions to rebuild a more adaptive world. Meaning found to help individuals cope with the traumas and grief over the loss of normalcy during challenging times. Wong (1989) found that personal meaning can endow life with personal significance, the coping helped to reduce psychological distress.

\section{METHODOLOGY}

Based on the literature review, the researcher goes through the constructs needed for meaningful life using an analogy to the work of Buheji and Ahmed (2020a,b) that focused on seeing and exploiting opportunities during a crisis or challenging times, similar to the situation caused by the COVID-19 pandemic. The framework of inspiration economy for exploitation of opportunities out of complex problems during unprecedented times is used as an analogy for meaning development. Sources of meaning development are illustrated to overcome the impairment caused by stressful events that caused anxiety. The inspiration economy framework is also used as meaning re-shaping and sharpening, or what one could call maintenance of meaning of life which is highly needed to alleviate the suffering waves during the crisis. The application of the framework is a means for developing models for improving the collective resilience during, and after the math of the COVID-19 pandemic and its related spillovers. 


\section{APPLICATION \& ANALYSIS}

\subsection{Complexity and Persistent Uncertainty During COVID-19 Pandemic}

Complexity recognises the uncertainty in all complex systems and their diversity. In a dynamic complex problem, multidisciplinary approaches could be a source of mental stimulation, which helps in dealing with uncertainty and capturing opportunities. Page (2010). Buheji et al. (2020a) confirmed that living in a status of uncertainty have increased a lot in the new normal. This uncertainty status needs to be met with clear and sharp meaning for life or existence if to be controlled from creating negative spillover.

The COVID-19 pandemic brought different complex issues relevant to life and livelihood. The number of these life and livelihood disrupting elements shack the 'meaning of existence' and 'hope in life'. Part of the unique complexity of the COVID-19 pandemic spillovers comes from the characteristics of the variety of the interacting elements and their level of interdependence, Page (2010). For example, the pandemic enforced social distancing, which reduced many services that were delivered based on engagement and made a sense of purpose.

With the continuation of the complex environment, even small decisions can have a negative effect on the individuals or the community if done without clear meaning in mind. Therefore, Buheji (2020d) sees that meaning for existence can help in sharpening the forecasting of the future, and effectively mitigating current and future risks.

\subsection{Role of Meaning in Absorbing Complexity}

Meaning can play a role in creating complex adaptive systems which ensure proper suitable interactions between the constructs of the different problems brought by the COVID-19 pandemic. When meaning is available for the defining moments we are going through; we can interpret the socioeconomic challenges and see them as an opportunity for social development or behavioural improvement. The new complexity perspectives that are mixed with meaning, prepare us for more for interventions or to be blended with the society besides preparing us more to be able to cope with and ethically accept the restrictions as part of the journey. Stillman et al. (2009).

Since complexity brings in usually diversity, and interdependence, the receiver of this complexity must have a broad and yet flexible thought, which can not be easily available except for those who have meaning and life purposefulness. When meaning is available, we can comprehend and create more acceptance of continuous change and its reality. Meaning of life purposefulness helps to create better coexistence without denying their multiplicity, randomness, and uncertainty, but seeking to coexist with them.

Without 'meaning of the living moment', we are more likely to fail to capture the profound knowledge of the rising complexity, which makes us more likely to go through the full topography of risks. The intervention of 'meaning of the moment' helps to convert the feeling of uncertainty into an acceptable risk that compensates with measures to be taken with the complex conditions. Buheji et al. (2021a) and Buheji et al. (2021b).

Our ability to relate to the meaning of the moment helps us to first be aware and then understanding the sensitivities of the change happening in our contemporary and future environment. This enhances our capacity to absorb the challenging changes in the context of the very large human, economic and ecological loss, and damage across the planet due to disruptions caused by the COVID-19 pandemic, Buheji (2020d). 


\subsection{Application of Inspiration Economy Framework for Exploiting Opportunities inside Complex Problems, Buheji and Ahmed (2020a).}

With the outbreak of the COVID-19 pandemic, the researcher published immediately in February (2020) a visualized framework that could help to mitigate the risks of this world emergency and turn it to be a source of a generation-defining moment where many contemporary and foresighted challenges or spillovers could be a source of bundle opportunities. However, over time and reflecting now a pandemic that lasted approximately now 22 months since the inception of the outbreak, one could reflect that this framework will not effectively work without having a receiver equipped with re-shaped or well-maintained 'meaning of the moment'. Buheji and Ahmed (2020a).

Figure (1) shows that both visible $(\mathrm{V})$ or hidden $(\mathrm{H})$ risks $(\mathrm{R})$ and opportunities $(\mathrm{O})$ are the source for contemporary $(\mathrm{C})$ and future foresight $(\mathrm{F})$ solutions. The different relations bring in low complexity low (CxL), or medium complexity $(\mathrm{CxM})$, or high complexity $(\mathrm{CxH})$. The work of Buheji and Ahmed (2020a) shown that multidisciplinary approaches (MDA's) are a must for dealing with such a framework, because it is one of the main mechanisms for coping with such an environment and turning it to be value-added 'generation-defining moment'.

The only proposed modification for the framework based on the latest work of Buheji and Ahmed (2021 and 2020) about the reasons for the 'untapped COVID-19 Solutions' is that 'meaning of the moment', as illustrated in Figure (1). With the availability of this moment, the framework would be more holistic and agile in dealing with the demands of a complex pandemic and its related spillovers.

Figure (1) Framework for Exploiting Opportunities inside Complex Problems equipment with the meaning of the living moment.

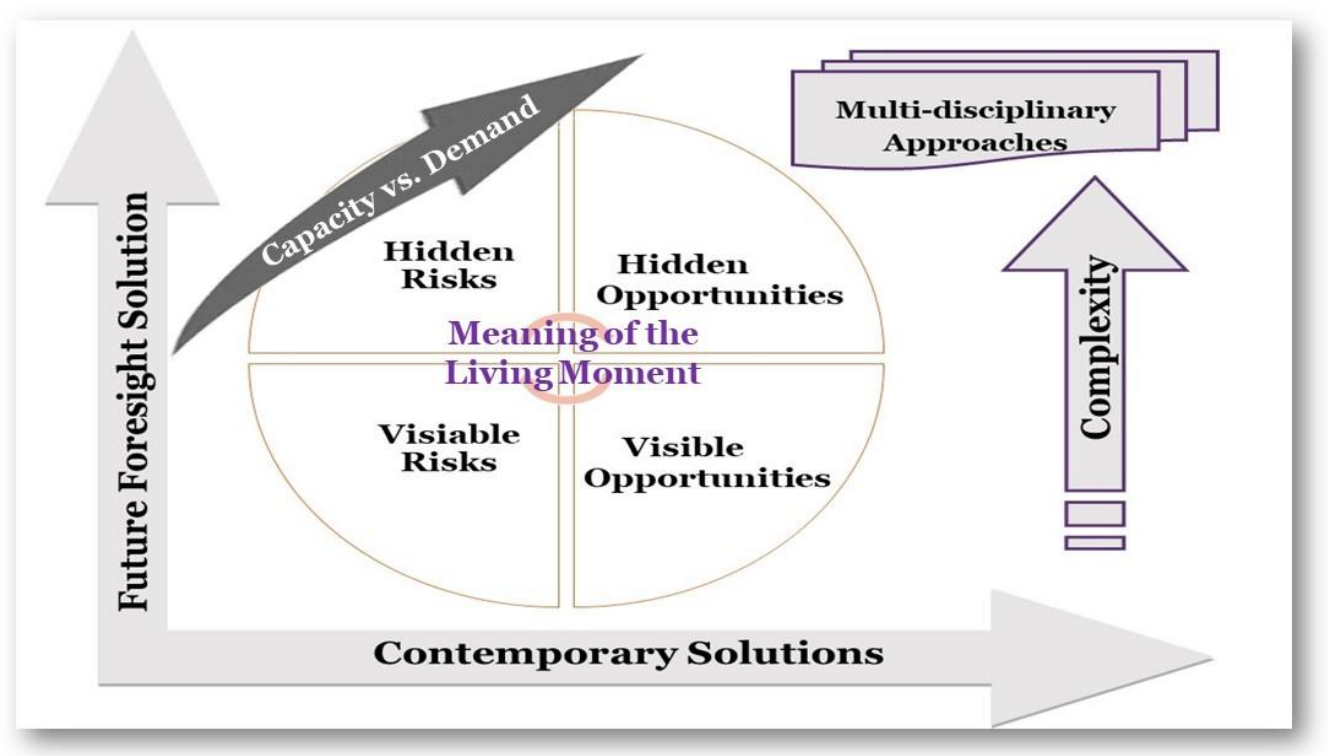

Figure 1 


\section{DISCUSSION AND CONCLUSION}

This paper reviews the meaning of living during unprecedented times and in a continuously complex world. The researcher reviews the coping mechanisms and the related working models that help to make sense of the world. More realisation of how the world is being challenged by complex problems, as the COVID-19 pandemic brought specific constraints. The author test whether these constraints are absorbed when the meaning of the moment is integrated within the receiver mindset.

The framework proposed shows that suffering due to a crisis similar to the COVID-19 pandemic could be mitigated when meaning and life purposefulness are renewed or sharpened. The contribution of this work is highly essential at this moment as it could help to mitigate the sharp rise of the collective trauma or the mental health problems due to the pandemic.

The recommendation here is that the proposed framework could be further stimulated with different cases where both visible and hidden opportunities and risks could be exploited. The measure and depth of meaning for the moment of life could be further correlated with both the opportunities or risks mitigation to understand what type of significance this could offer, Angeli and Montefusco (2020), Buheji and Ahmed (2020b). This work also could help to ease current and coming generations burdensome of the foresighted future that is full of instability, inherent unpredictability, and uncertainty, besides being more complex. The influence of meaning of the moment could be like a chip, once embedded, could enhance credible diversity and raise the human capacity to positively interpret any condition with high resilience and tolerance. This is the basis of survival. Buheji et al. (2020a) and Buheji et al. (2020b).

Without 'meaning of the living moment', we are more likely to fail to capture the profound knowledge of the rising complexity, making us more likely to go through the entire topography of risks.

\section{REFERENCES}

[1] Allen, P., Maguire, S., \& McKelvey, B. (2011). The SAGE Handbook of Complexity and Management.

[2] Angeli, F., \& Montefusco, A. (2020). Sensemaking and learning during the Covid-19 pandemic: A complex adaptive systems perspective on policy decision-making. World Development, 136, 105106. https://doi.org/10.1016/j.worlddev.2020.105106

[3] Arslan, G., and Allen, K. (2021). Exploring the association between coronavirus stress, meaning in life, psychological flexibility, and subjective wellbeing. Psychol. Health Med. doi: $10.1080 / 13548506.2021 .1876892$

[4] Arslan, G., and Yıldırım, M. (2021). Coronavirus stress, meaningful living, optimism, and depressive symptoms: a study of moderated mediation model. Austr. J. Psychol. doi: $10.1080 / 00049530.2021 .1882273$

[5] Arslan G, Y1ldırım M and Leung MM (2021) Mediating Effect of Personal Meaning in the Prediction of Life Satisfaction and Mental Health Problems Based on Coronavirus Suffering. Front. Psychol. 12:638379. doi: 10.3389/fpsyg.2021.638379

[6] Baumeister, R. F., Vohs, K. D., Aaker, J. L., and Garbinsky, E. N. (2013). Some key differences between a happy life and a meaningful life. J Posit. Psychol. 8, 505-516. doi: $10.1080 / 17439760.2013 .830764$

[7] Buheji, M (2020a) Coronavirus as a Global Complex Problem Looking for Resilient Solutions, Business Management and Strategy, Vol. 11, No. 1, 94-109. 
[8] Buheji, M (2020b) Mitigating Covid-19 Pandemic Spillovers on Suicidal Behaviours - The Second Wave, International Journal of Management (IJM) Volume 11, Issue 11, November 2020, pp. 1757-1771.

[9] Buheji, M (2020c) Psychological Resilience and Poor Communities Coping with COVID-19 Pandemic, International Journal of Psychology and Behavioral Sciences, Vol. 10 No. 4, pp. 100-108.

[10] Buheji, M (2020d) Insights - Thoughts Pioneering the Future of Our Socio-Economies, especially post-COVID-19 pandemic. Researchgate Self-Publish, (Published in Sep).

[11] Buheji, M (2020e) Optimising 'Moments of Pauses' - A Reflections from COVID-19 Pandemic, International Journal of Psychology and Behavioral Sciences, Vol. 10 No. 4, 2020, pp. 85-92.

[12] Buheji, M. (2017) Understanding Mechanisms of Resilience Economy- Live Application on a Complex Business Model. Advances in Social Sciences Research Journal, 4(14), pp. 52-64.

[13] Buheji, M; Ahmed, D (2021) 'Covid-19 The Untapped Solutions' (Part Two), Westwood Books Publishing LLC, USA.

[14] Buheji, M and Ahmed, D (2020a) Foresight of Coronavirus (COVID-19) Opportunities for a Better World, American Journal of Economics; 10(2): 97-108.

[15] Buheji, M; Ahmed, D (2020b) 'Covid-19 The Untapped Solutions' (Part One), Westwood Books Publishing LLC, USA.

[16] Buheji, M and Jahrami, H (2020) Analysing Hardiness Resilience in Covid-19 Pandemic Using Factor Analysis, International Journal of Management, Volume 11, Issue 10, Oct 2020, pp. 802-815.

[17] Buheji, M; Ahmed, D and Jahrami, H (2020a) Living Uncertainty in the New Normal, International Journal of Applied Psychology; 10(2): 21-31.

[18] Buheji, M; Mavrić, B; Beka, G; Yein, T (2020b) Alleviation of Refugees COVID-19 Pandemic Risks- A Framework for Uncertainty Mitigation, International Business Research, Vol. 13, No. 7, pp. 69-79.

[19] Buheji, M; Jahrami, H; Dhahi, A (2020c) Minimising Stress Exposure During Pandemics Similar to COVID-19, International Journal of Psychology and Behavioral Sciences, Vol. 10 No. 1, pp. 9-16.

[20] da Silva. M; Rocha, R; Buheji, M; Jahrami, H and Cunha, K (2020) A systematic review of the prevalence of anxiety symptoms during coronavirus epidemics, Journal of Health Psychology, pp. $1-11$

[21] de Jong, E. M., Ziegler, N., and Schippers, M. C. (2020). From shattered goals to meaning in life: life crafting in times of the COVID-19 pandemic. Front. Psychol. 11:577708. doi: 10.3389/fpsyg.2020.577708

[22] Edwards, M., and Van Tongeren, D. R. (2020). Meaning mediates the association between suffering and wellbeing. J. Posit. Psychol. 15, 722-733. doi: 10.1080/17439760.2019.1651890

[23] Frankl, V. E. (1985). Man's Search for Meaning. New York, NY: Washington Square Press. 
[24] Heine, S. J., Proulx, T., and Voh, K. D. (2006). The meaning maintenance model: on the coherence of social motivations. Pers. Soc. Psychol. Rev. 10, 88-110. doi: $10.1207 / \mathrm{s} 15327957$ pspr1002_1

[25] Heintzelman, S. J., and King, L. A. (2014). Life is pretty meaningful. Am. Psychol. 69, 561574. doi: 10.1037/a0035049

[26] Hooker, S. A., Masters, K. S., Vagnini, K. M., and Rush, C. L. (2020). Engaging in personally meaningful activities is associated with meaning salience and psychological wellbeing. J. Posit. Psychol. 15, 821-831. doi: 10.1080/17439760.2019.1651895

[27] Ho, M. Y., Cheung, F. M., and Cheung, S. F. (2010). The role of meaning in life and optimism in promoting wellbeing. Pers. Indiv. Dif. 48, 658-663. doi: 10.1016/j.paid.2010.01.008

[28] King, L. A., Hicks, J. A., Krull, J. L., and Del Gaiso, A. K. (2006). Positive affect and the experience of meaning in life. J. Person. Soc. Psychol. 90, 179-196. doi: 10.1037/00223514.90.1.179

[29] Martela, F., and Steger, M. (2016). The three meanings of meaning in life: Distinguishing coherence, purpose, and significance. J. Posit. Psychol. 11, 531-545. doi: $10.1080 / 17439760.2015 .1137623$

[30] Page, S. (2010). Diversity and Complexity. Princeton University Press.

[31] Park, C. L. (2010). Making sense of the meaning literature: an integrative review of meaning making and its effects on adjustment to stressful life events. Psychol. Bull. 136, 257-301. doi: $10.1037 / \mathrm{a} 0018301$

[32] Schnell, T., and Krampe, H. (2020). Meaning in life and self-control buffer stress in times of COVID-19: moderating and mediating effects with regard to mental distress. Front. Psychiatry 11:582352. doi: $10.3389 /$ fpsyt.2020.582352

[33] Steger, M. F., and Frazier, P. (2005). Meaning in life: one link in the chain from religiousness to wellbeing. J. Couns. Psychol. 52, 574-582. doi: 10.1037/0022-0167.52.4.574

[34] Stillman, T. F., Baumeister, R. F., Lambert, N. M., Crescioni, A. W., DeWall, C. N., and Fincham, F. D. (2009). Alone and without purpose: life loses meaning following social exclusion. J. Exp. Soc. Psychol. 45, 686-694. doi: 10.1016/j.jesp.2009.03.007

[35] Testoni, I., Sansonetto, G., Ronconi, L., Rodelli, M., Baracco, G., and Grassi, L. (2018). Meaning of life, representation of death, and their association with psychological distress. Palliat. Support. Care 16, 511-519. doi: 10.1017/S1478951517000669

[36] Trzebiński, J., Caba?ski, M., and Czarnecka, J. C. (2020) Reaction to the COVID-19 pandemic: the influence of meaning in life, life satisfaction, assumptions on world orderliness and positivity. J. Loss Trauma 25, 544-557. doi: 10.1080/15325024.2020.1765098

[37] Van Tongeren, D. R., and Green, J. D. (2010). Combating meaninglessness: on the automatic defense of meaning. Pers. Soc. Psychol. Bull. 36, 1372-1384. doi: 10.1177/0146167210383043

[38] Van Tongeren, D. R., and Green, J. D. (2018). Meaning and death-thought accessibility. Br. J. Soc. Psychol. 57, 230-239. doi: 10.1111/bjso.12212 
[39] Van Tongeren, D. R., Green, J. D., Hook, J. N., Davis, D. E., Davis, J. L., and Ramos, M. (2015). Forgiveness increases meaning in life. Soc. Psychol. Pers. Sci. 6, 47-55. doi: $10.1177 / 1948550614541298$

[40] Van Tongeren DR and Showalter Van Tongeren SA (2021) Finding Meaning Amidst COVID19: An Existential Positive Psychology Model of Suffering. Front. Psychol. 12:641747. doi: $10.3389 /$ fpsyg. 2021.641747

[41] VanderWeele T. (2020) Challenges Estimating Total Lives Lost in COVID-19 Decisions: Consideration of Mortality Related to Unemployment, Social Isolation, and Depression. JAMA; 324(5):445-446.

[42] Wong, P. T. P. (2016). "Meaning-seeking, self-transcendence, and wellbeing," in Logotherapy and Existential Analysis: Proceedings of the Viktor Frankl Institute, Vol. 1, ed A. Batthyany (Cham: Springer), 311-322. doi: 10.1007/978-3-319-29424-7_27

[43] Wong, P. T. P. (1998). "Spirituality, meaning, and successful aging," in The Human Quest for Meaning: A Handbook of Psychological Research and Clinical Applications, eds P. T. P. Wong and P. Fry (Mahwah, NJ: Lawrence Erlbaum Associates, Inc.), 359-394.

[44] Yildirim, M., Arslan, G., and Wong, P. T. P. (2020). Meaningful living, resilience, affective balance, and psychological health problems during COVID-19. Curr. Psychol. doi: 10.1007/s12144-020-01244-8 